\title{
MEASURES OF IRREGULARITY OF GRAPHS
}

\author{
Joelma Ananias de Oliveira ${ }^{1}$, Carla Silva Oliveira ${ }^{2}$, \\ Claudia Justel ${ }^{3}$ and Nair Maria Maia de Abreu ${ }^{4 *}$
}

Received June 13, 2012 / Accepted March 15, 2013

\begin{abstract}
A graph is regular if every vertex is of the same degree. Otherwise, it is an irregular graph. Although there is a vast literature devoted to regular graphs, only a few papers approach the irregular ones. We have found four distinct graph invariants used to measure the irregularity of a graph. All of them are determined through either the average or the variance of the vertex degrees. Among them there is the index of the graph, a spectral parameter, which is given as a function of the maximum eigenvalue of its adjacency matrix. In this paper, we survey these invariants with highlight to their respective properties, especially those relative to extremal graphs. Finally, we determine the maximum values of those measures and characterize their extremal graphs in some special classes.
\end{abstract}

Keywords: index of a graph, irregularity measure, extremal graphs.

\section{INTRODUCTION}

Let $G$ be an undirected graph with $n$ vertices and $m$ edges without loops and multiple edges. We denote $\mathcal{G}(n)$ and $\mathcal{H}(n)$, the respective sets of all the graphs (connected or not) and all the connected graphs with $n$ vertices and, $\mathcal{G}(n, m)$ and $\mathcal{H}(n, m)$, the respective sets of the graphs (connected or not) and all the connected graphs with $n$ vertices and $m$ edges. The degree of a vertex $v_{i}$, denoted $d\left(v_{i}\right)$, is the number of the incident edges in $v_{i}$. Since $\sum_{i=1, n} v_{i}=2 m$, the average of vertex degrees can be given as $\bar{d}(G)=\frac{2 m}{n}$. A graph is $k$-regular or, simply regular, if every degree is equal to $k$. Otherwise, the graph is said to be an irregular graph.

The eigenvalues of a graph are the eigenvalues of its adjacency matrix. They are also respectively referred as the $A$-eigenvalue, denoted $\lambda_{1} \geq \lambda_{2} \geq \ldots \geq \lambda_{n}$. The index of a graph is the maximal eigenvalue of $A(G)$, that is, $\lambda_{1}(G)$. The join $G_{1} \vee G_{2}$ of (disjoint) graphs $G_{1}$ and $G_{2}$ is the graph obtained from $G_{1}$ and $G_{2}$ by joining each vertex of $G_{1}$ with each vertex of $G_{2}$.

\footnotetext{
*Corresponding author

${ }^{1}$ Universidade Federal de Mato Grosso, Brasil. E-mail: jo.ananias@ gmail.com

${ }^{2}$ Escola Nacional de Ciências Estatísticas, Rio de Janeiro, Brasil. E-mail: carla.oliveira@ibge.gov.br

3 Instituto Militar de Engenharia, Rio de Janeiro, Brasil. E-mail: cjustel@ime.eb.br

${ }^{4}$ Universidade Federal do Rio de Janeiro, Brasil. E-mail: nair@pep.ufrj.br
} 
Given a graph $G$, what is the minimal number of edges needed to add to $G$ to get $H$, a regular supergraph of $G$ ? In this case, which pairs of non adjacent vertices in $G$ should become adjacent in $H$ ? Questions such as these are interesting and in order to try to answer them it seems useful to know how near or distant any graph is from a regular one. We define as the measure of irregularity of $G$, every function $F: G \mapsto \mathbb{R}$, where $G$ is the set of all graphs and $\mathbb{R}$ is the set of all real number, such that $\forall G \in G, G$ is a regular graph if and only if $F(G)=0$.

In this paper, we survey all known parameters used as measures of irregularity as well as their respective properties, mainly those relative to upper and lower bounds and their respective extremal graphs. Also, we characterize their extremal graphs among all the split complete graphs and among all path complete graphs with a particular characteristic.

\section{KNOWN MEASURES OF IRREGULARITY}

In 1957, Collatz \& Sinogowitz [8] proved that the index of a graph is greater than or equal to the average of vertex degrees and the equality holds if and only if the graph is regular. From this fact, they introduced $\varepsilon(G)=\lambda_{1}(G)-\bar{d}(G)$ to measure the irregularity of a graph, which we call here, the spectral measure. Those authors also proved that, for $n \leq 5$, the maximum value to $\varepsilon(G)$ is $\sqrt{n-1}-2+\frac{2}{n}$ and the maximal is attained for the star $S_{n}$. Based on this, they conjectured that $S_{n}$ is the most irregular graph among all graphs with $n$ vertices relative to $\varepsilon(G)$. In 1988 , this conjecture was refuted by Cvectković \& Rowlinson [9].

Some years after, Bell [4] suggested making the variance of the degrees of the vertices of $G$,

$$
\sigma(G)=\frac{1}{n} \sum_{i=1}^{n}\left(d\left(v_{i}\right)\right)^{2}-\frac{1}{n^{2}}\left(\sum_{i=1}^{n} d\left(v_{i}\right)\right)^{2},
$$

as a measure of the irregularity of $G$, and we refer to it as the variance measure. Bell characterized the most irregular graphs in the classes $\mathcal{G}(n), \mathcal{G}(n, m)$ and $\mathcal{H}(n, m)$ concerning the both measures $\varepsilon(G)$ and $\sigma(G)$. In the same paper, he obtained upper and lower bounds of $\sigma(G)$ as functions of $n$ and $m$.

Albertson [2] defined the imbalance of an edge $\left(v_{i}, v_{j}\right)$ by $i m b_{v_{i}} v_{j}=\left|d\left(v_{i}\right)-d\left(v_{j}\right)\right|$ and he used it to introduce $\operatorname{irr}(G)=\sum_{\left(v_{i}, v_{j}\right) \in E} i m b_{v_{i} v_{j}}$ as a measure of the irregularity of $G$ and, in the same paper, $\operatorname{irr}(G)<\frac{4 n^{3}}{27}$ is proven. In 2000, Hansen et al. [10] presented a bound to $\operatorname{irr}(G)$ as a function of $n$ and $m$ and determined extremal graphs for it. Here, we refer this invariant as the imbalance measure.

More recently, Nikiforov [13] introduced $s(G)=\sum_{v_{i} \in V(G)}\left|d\left(v_{i}\right)-\frac{2 m}{n}\right|$ as a new measure of the irregularity of a graph. Also, he showed several inequalities with respect to $s(G), \varepsilon(G)$ and $\sigma(G)$. The measure of Nikiforov will be named by the degree deviation measure.

It is easy to see that $\varepsilon(G), \sigma(G)$ and $s(G)$ can be taken by $F(G)$, since $\varepsilon(G)=\sigma(G)=s(G)=$ 0 if and only if $G$ is a regular graph. Although $\operatorname{irr}(G)$ is considered in the literature as an irregularity measure, it can be null even when $G$ is an irregular graph. In this case, $\operatorname{irr}(G)=0$ if 
and only if $G$ is either regular or a disconnected graph with all the regular components. Figure 1 illustrates this fact.

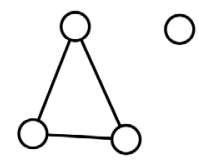

$\mathrm{G}_{1}$

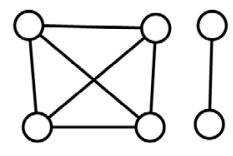

$\mathrm{G}_{2}$
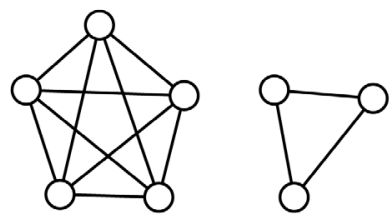

Figure 1 - Disconnected graphs such that $\operatorname{irr}\left(G_{1}\right)=\operatorname{irr}\left(G_{2}\right)=\operatorname{irr}\left(G_{3}\right)=0$.

\section{EXTREMAL IRREGULARITY IN GENERAL CLASSES OF GRAPHS}

This section is devoted to define the families of graphs where some elements are extremal for $\varepsilon(G), \sigma(G)$ and $\operatorname{irr}(G)$ where $G$ is a graph belonging to one of the most general classes such as $\mathcal{G}(n), \mathcal{H}(n), G(n, m)$ and $\mathcal{H}(n, m)$. Then, theorems are presented to show the conditions under which those graphs are extremal for the measures studied. The section ends with a table summarizing these results.

Definition 3.1. [1] A graph with $n$ vertices and $m$ edges is a quasi-complete graph, denoted $Q C(n, m)$, when it is obtained as follows:

(i) its vertices are labeled $1, \ldots, n$;

(ii) the integers $d$ and $t, 2 \leq d, 0 \leq t<d$, are defined such that $m=\left(\begin{array}{l}d \\ 2\end{array}\right)+t$;

(iii) the vertices labeled $1, \ldots, d$ form the maximal clique;

(iv) the vertex $d+1$ is adjacent to $t$ vertices labeled $1, \ldots, t$ and the $n-(d+1)$ remaining vertices are isolated.

Figure 2 displays some examples of quasi-complete graphs.

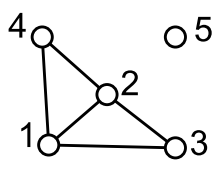

QC $(5,5)$

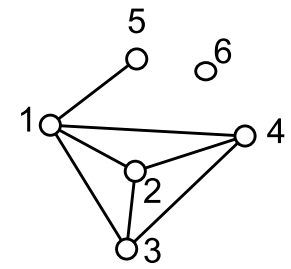

$\mathrm{QC}(6,7)$

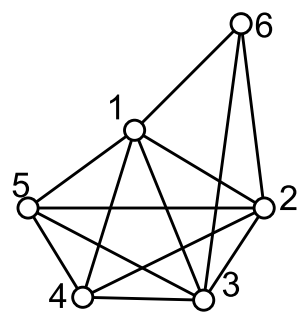

QC $(6,13)$

Figure 2 - Graphs $Q C(5,5), Q C(6,7)$ and $Q C(6,13)$. 
The first result, due to Bell [4], gives the maximum value of the spectral measure, $\varepsilon(G)$, where $G$ is a graph (not necessarily connected) with $n$ vertices. Moreover, it shows that the extremal graphs to $\varepsilon(G)$ are the quasi-complete graphs. Proposition 3.2, also due to Bell, proves that among all graphs with $n$ vertices and $m$ edges, no necessarily connected, the quasi-complete graph $Q C(n, m)$ is the only one that satisfies the maximal value of the spectral measure.

Proposition 3.1. [4] Given $n$, write $m=\left(\begin{array}{c}\left\lfloor\frac{1}{2}(n+1)\right\rfloor \\ 2\end{array}\right)$ and $m^{\prime}=\left(\begin{array}{c}\left\lfloor\frac{1}{2}(n+1)\right\rfloor+1 \\ 2\end{array}\right)$. Then, we have

$$
\max \{\varepsilon(G): G \in G(n)\}=\left\{\begin{array}{l}
\frac{1}{4} n-\frac{1}{2}(n \text { even }) \\
\frac{1}{4} n-\frac{1}{2}+\frac{1}{4 n}(n \text { odd }) .
\end{array}\right.
$$

This maximum is attained uniquely by $Q C(n, m)$ if $n$ is odd, and by $Q C(n, m)$ and $Q C\left(n, m^{\prime}\right)$ (only) if $n$ is even.

For example, if $n=6, \max \{\varepsilon(G): G \in G(6)\}=1$ and $G$ is either $Q C(6,3)=K_{3} \cup 3 K_{1}$ or $Q C(6,6)=K_{4} \cup 2 K_{1}$.

Proposition 3.2. [4] Let $n$ and $m$ be given with $m \leq\left(\begin{array}{l}n \\ 2\end{array}\right)$. Then, $\max \{\varepsilon(G): G \in G(n, m)\}$ is attained uniquely by $Q C(n, m)$.

The following result gives the maximum value of the variance measure, $\sigma(G)$, when $G$ is a graph with $n$ vertices, and it proves that the maximum is attained where $G$ is a quasi-complete graph.

Proposition 3.3. [4] Given n, write $r=\left\lfloor\frac{1}{4}(3 n+2)\right\rfloor$ and $m=\left(\begin{array}{c}r \\ 2\end{array}\right)$. Then, we have $\max \{\sigma(G)$ : $G \in G(n)\}=\frac{r}{n^{2}}(r-1)^{2}(n-r)$, and this maximum is attained by $Q C(n, m)$.

For example, if $n=7, \max \{\sigma(G): G \in G(7)\}=3,26$ and $G$ is $Q C(7,10)=K_{5} \cup 2 K_{1}$.

For specific values of $n$ and $m$, the quasi-star graphs, defined as follows, give the extremal graph to the variance measure in the class of the connected graphs with $n$ vertices.

Definition 3.2. [1] A quasi-star, denoted $Q S(n, m)$, is a graph with $n$ vertices and $m$ edges when it is obtained as follows:

(i) its vertices are labeled $1, \ldots, n$;

(ii) the integers $d$ and $t, 2 \leq d, 0 \leq t<d$, are defined such that $m=\left(\begin{array}{l}n \\ 2\end{array}\right)-\left(\begin{array}{l}d \\ 2\end{array}\right)-t$;

(iii) the first $n-d-1$ vertices are adjacent to all others vertices of the graph;

(iv) the vertex $n-d$ is adjacent to vertices labeled $1, \ldots, n-t$.

The Figure 3 shows quasi-star graphs with 5 and 6 vertices.

The next result is an immediate consequence of Proposition 3.4 and it was presented in [4] as its corollary. Proposition 3.5 shows that among all graphs with $n$ vertices and $m$ edges, the extremal 


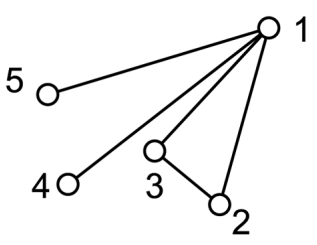

QS $(5,5)$

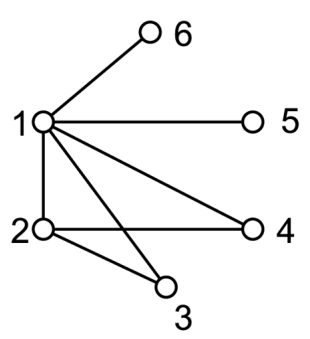

QS $(6,7)$

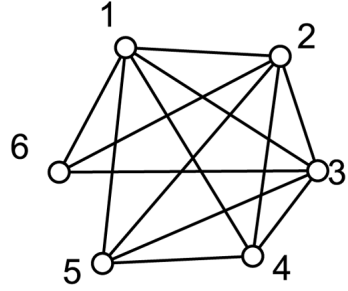

QS $(6,13)$

Figure 3 - Graphs $Q S(5,5), Q S(6,7)$ and $Q S(6,13)$.

ones relative to the variance measure are quasi-stars $Q S(n, m)$, when they are sparse graphs; otherwise, the extremal graphs are quasi-complete $Q C(n, m)$.

Proposition 3.4. [4] Given $n$, write $r=\left\lfloor\frac{1}{4}(3 n+2)\right\rfloor$ and $m=\left(\begin{array}{l}n \\ 2\end{array}\right)-\left(\begin{array}{l}r \\ 2\end{array}\right)$. Then, we have $\max \{\sigma(G): G \in \mathcal{H}(n)\}=\frac{r}{n^{2}}(r-1)^{2}(n-r)$, and this maximum is attained by $Q S(n, m)$.

For example, if $n=7, \max \{\sigma(G): G \in \mathcal{H}(7)\}=3,26$ and $G$ is $Q S(7,11)=K_{1} \vee K_{1,5}$.

Proposition 3.5. [4] Let $n$ and $m$ be given, with $m \leq\left(\begin{array}{l}n \\ 2\end{array}\right)$. Then, the $\max \{\sigma(G): G \in G(n, m)\}$ is attained by $Q C(n, m)$ if $m>\frac{1}{2}\left(\begin{array}{c}n \\ 2\end{array}\right)+\frac{n}{2}$, and by $Q S(n, m)$ if $m<\frac{1}{2}\left(\begin{array}{c}n \\ 2\end{array}\right)-\frac{n}{2}$.

For example, if $n=6$ and $m=11, \max \{\sigma(G): G \in \mathcal{H}(6,11)\}=1,55$ and $G$ is $Q C(6,11)=$ $K_{1} \vee\left(K_{4} \cup K_{1}\right)$. If $n=6$ and $m=4, \max \{\sigma(G): G \in \mathcal{H}(6,4)\}=1,55$ and $G$ is $Q S(6,4)=$ $S_{5} \cup K_{1}$.

Definition 3.3. [7] Let $0 \leq k \leq n-3$ and let $H_{n, k}$ be a connected graph with $n+k$ edges. $H_{n, k}$ is obtained as follows:

(i) label the vertices from 1 to $n$;

(ii) add $n-1$ edges from the vertex labeled 1 to $2, \ldots, n$ (a star $S_{n}$ with center in 1$)$;

(iii) add $k$ edges from the vertex 2 to each vertex $3, \ldots, k+3$.

The graphs $H_{n, k}$ result from the following join $H_{n, k}=K_{1} \vee\left(K_{1, k+1} \cup \bar{K}_{n-k-3}\right)$. Similar to them, Definition 3.4 introduces the graphs $G_{n, k}$. For a large enough $n$, the first graphs satisfy the maximum value to the spectral measure among all connected graphs with $n$ vertices and $n+k$ edges; the second graphs satisfy the maximum values of the variance measure among all non sparse connected graphs with $n$ vertices. Some examples of graphs $H_{n, k}$ are shown in Figure 4.

Proposition 3.6. [4] For fixed $k \geq 3$, and $n$ sufficiently large (depending on $k$ ), $\max \{\varepsilon(G): G \in$ $\mathcal{H}(n, n+k)\}$ is attained uniquely by $H_{n, k}$. 

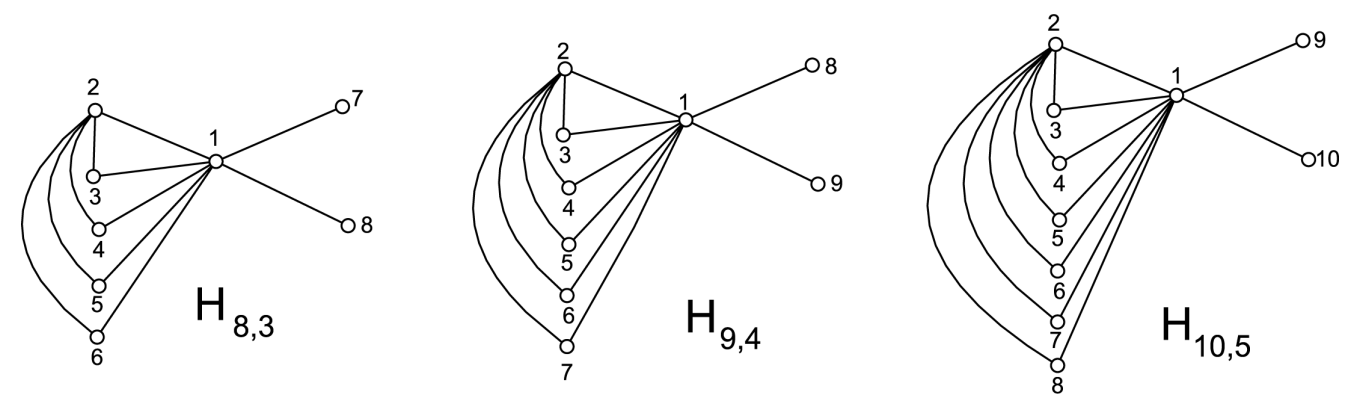

Figure 4 - Graphs $H_{8,3}, H_{9,4}$ and $H_{10,5}$.

Definition 3.4. [9] For $k \in \mathbb{Z}$ such that $0 \leq k<\frac{n(n-3)}{2}, G_{n, k}$ results of the join of $K_{1}$ and $Q C(n-1, k)$, that is, $G_{n, k}=K_{1} \vee Q C(n-1, k)$.

See some examples of graphs $G_{n, k}$ in the Figure 5.
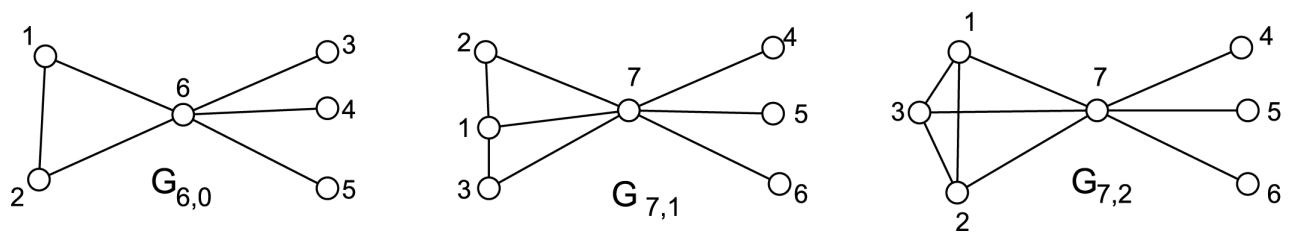

Figure 5 - Graphs $G_{6,0}, G_{7,1}$ and $G_{7,2}$.

Proposition 3.7. [4] If $n$ and $m=n+k$ are such that $n-1 \leq m \leq\left(\begin{array}{l}n \\ 2\end{array}\right)$, then $\max \{\sigma(G): G \in$ $\mathcal{H}(n, m)\}$ is attained by $G_{n, k}$ if $m>\frac{1}{2}\left(\begin{array}{l}n \\ 2\end{array}\right)+n-1$, and by $Q S(n, m)$ if $m<\frac{1}{2}\left(\begin{array}{l}n \\ 2\end{array}\right)$.

Definition 3.5. [3] Given $n$ and $q$ integers such that $0 \leq q \leq n$, a pineapple graph, denoted by $P A(n, q)$, is a graph with $n$ vertices consisting of a clique on $q$ vertices and a stable set on the remaining $n-q$ vertices in which each vertex of the stable set is adjacent to a unique and the same vertex of the clique.

Aouchiche et al., in [3], conjectured that, among all connected graphs with $n$ vertices, the maximal relative to the spectral measure are the pineapple graphs such that the size of the maximal clique is one or two unities more than the size of the maximal stable set.

Conjecture 3.1. [3] The most irregular graph to $\varepsilon(G)$, where $G$ is a connected graph on $n$ $(n \geq 10)$ vertices, is a pineapple PA(n,q)) in which the clique size q is equal to $\left\lfloor\frac{n}{2}\right\rfloor+1$.

Finally, it follows the definition of the family of graphs which are maximal to the imbalance measure.

Definition 3.6. [3] Given $n, k$ and $t$ integers such that $t \leq k \leq n$, a fanned complete split graph, denoted by $\operatorname{FCS}(n, k, t)$, is a graph with $n$ vertices obtained from a complete split graph $C S(n, k)$ by connecting a vertex from the stable set by edges to tother vertices of the stable set. 
Theorem 3.1. [10] For any graph $G$ with $n$ vertices, $m$ edges and irregularity irr $(G)$,

$$
\operatorname{irr}(G) \leq k(n-k)(n-k-1)+t(t-2 k-1)
$$

where

$$
k=\left\lfloor n-\frac{1}{2}-\sqrt{\left(n-\frac{1}{2}\right)^{2}-2 m}\right\rfloor
$$

and

$$
t=m-(n-k) k-k(k-1) / 2 .
$$

Moreover, this value is attained if and only if $G$ is a fanned complete split graph.

The Figure 6 shows the extremal fanned complete split graph with 8 vertices and 15 edges for imbalance measure, where $\operatorname{irr}(F C S(8,2,2))=54$.
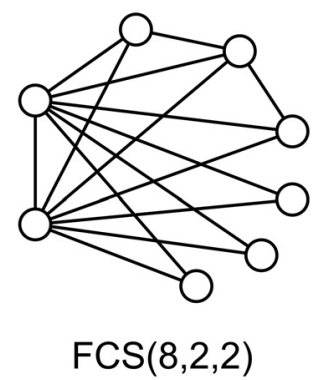

Figure 6 - Graph $\operatorname{FCS}(8,2,2)$.

Table 1 shows, in each line of the third column, the extremal graph among all graphs in the class on the correspondent line of the second column. Each extremal graph is relative to the irregularity measure displayed in the same line of the first column. The last column gives the references that support those results.

\section{EXTREMAL IRREGULARITY IN $P C_{n, p, 1}$ AND $S C(n, k)$ GRAPHS}

The results presented in this section come originally from [14] and approach the following connected graphs with order $n$ : the graphs $P C_{n, p, 1}=\overline{K_{1, n-p-1} \cup p K_{1}}$, which are special case of path complete graphs (see Definition 4.1, [5], [12]) and the complete split graphs $S C(n, k)$, special cases of the well known split graphs, (see Definition 4.2 and [6]).

Definition 4.1. Let $n, m, p, t \in \mathbb{N}$, with $1 \leq t \leq n-2$ and $1 \leq p \leq n-t-1$. A graph withn vertices and $m$ edges such that

$$
\frac{(n-t)(n-t-1)}{2}+t \leq m \leq \frac{(n-t)(n-t-1)}{2}+n-2
$$

is called path-complete graph, denoted $P C_{n, p, t}$, if and only if 


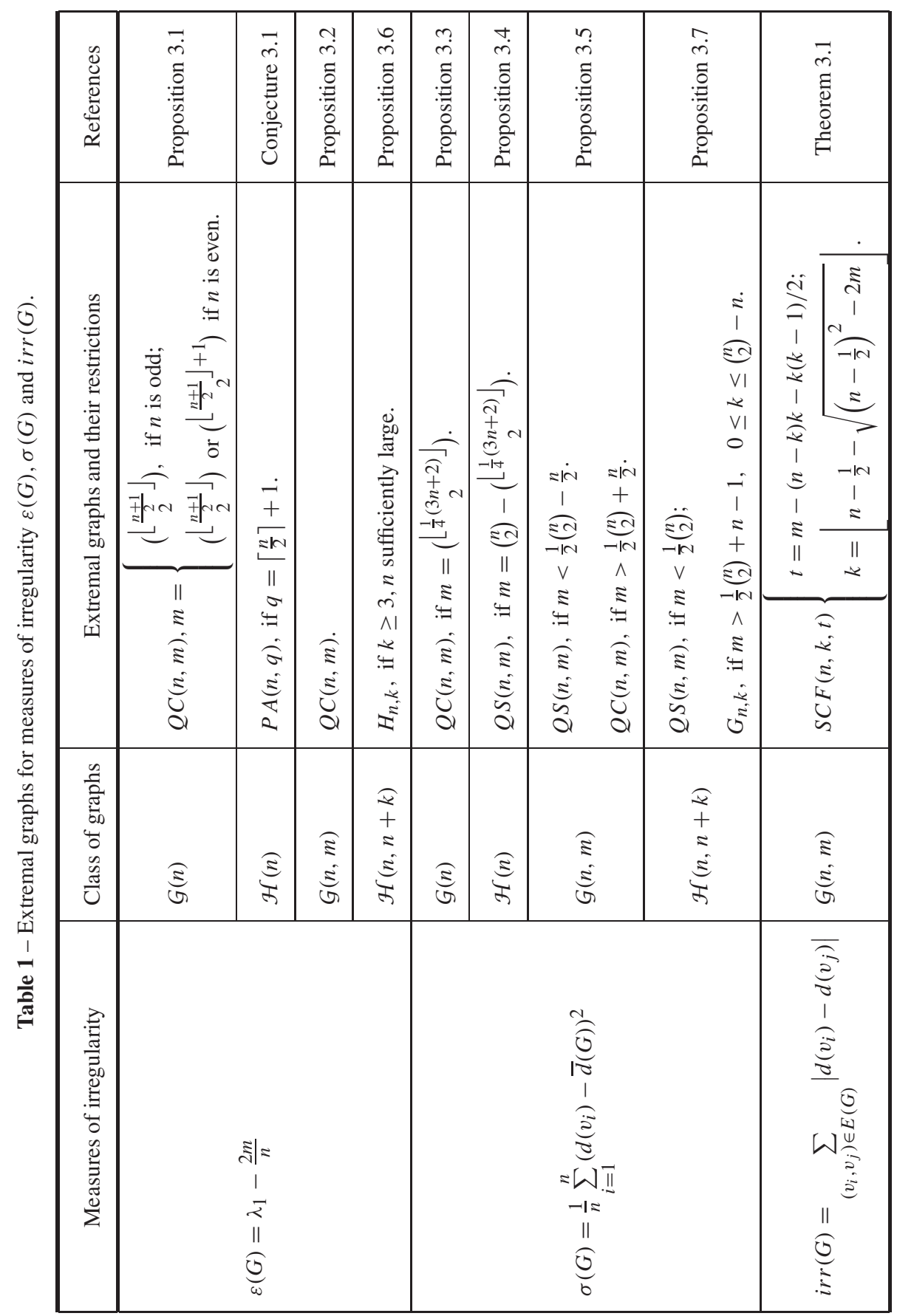


(i) the maximal clique of $P C_{n, p, t}$ is $K_{n-t}$;

(ii) $P C_{n, p, 1}$ has a t-path $P_{t+1}=\left[v_{0}, v_{1}, v_{2}, \ldots, v_{t}\right]$ such that $v_{0} \in K_{n-t} \cap P_{t+1}$ and $v_{1}$ is joined to $K_{n-t}$ by $p$ edges;

(iii) there are no other edges.

In the case where $t=1$, we have $P C_{n, p, 1}$ is also a split graph. The first two theorems give lower and upper bounds to the degree deviation and the variance measures of $P C_{n, p, 1}$. Moreover, it characterize extremal graphs that attained those bounds. The third theorem gives the lower and upper bounds to the imbalanced measure.

Theorem 4.1. For $n \geq 4$ and $\forall p, 1 \leq p \leq n-2$, we have $\frac{4(n-2)}{n} \leq s\left(P C_{n, p, 1}\right) \leq \frac{2(n-2)^{2}}{n}$. The lower bound is attained by $P C_{n, n-2,1}$ and the upper bound is attained by $P C_{n, 1,1}$.

Proof. Let $P C_{n, p, 1}$ and $P C_{n, p+1,1}$ be two path complete graphs. Through a simple algebraic manipulation, we obtain

$$
s\left(P C_{n, p, 1}\right)-s\left(P C_{n, p+1,1}\right) \geq \frac{(n-p-1)(2 n-4)}{n}+\frac{(n-p-2)(-2 n+4)}{n} .
$$

Consequently, $s\left(P C_{n, p, 1}\right)-s\left(P C_{n, p+1,1}\right) \geq \frac{2 n-4}{n}$. Since $n>2, s\left(P C_{n, p, 1}\right)-s\left(P C_{n, p+1,1}\right) \geq$ 0 , we conclude that $\forall p, 1 \leq p \leq n-3, s\left(P C_{n, p, 1}\right)>s\left(P C_{n, p+1,1}\right)$.

Theorem 4.2. For $n \geq 4$ and $1 \leq p \leq n-2$, we have $\frac{2(n-2)}{n^{2}} \leq \sigma\left(P C_{n, p, 1}\right) \leq \frac{(n-2)\left(n^{2}-5 n+8\right)}{n^{2}}$. The lower bound is attained by $P C_{n, n-2,1}$ and the upper bound by $P C_{n, 1,1}$.

Proof. From the definition of the variance measure, it is easy to reach $P C_{n, p, 1}$ as follows,

$$
\sigma\left(P C_{n, p, 1}\right)=\frac{(n-p-1)[(n-4)(n-p)+4]}{n^{2}} .
$$

So, we have, $\sigma\left(P C_{n, p, 1}\right)-\sigma\left(P C_{n, p+1,1}\right)=\frac{2(n-p-1)(n-4)+4}{n^{2}}>0$. Since the difference above is strictly positive, we obtain $\forall p, 1 \leq p \leq n-3, \sigma\left(P C_{n, p, 1}\right)>\sigma\left(P C_{n, p+1,1}\right), \sigma\left(P C_{n, 1,1}\right)=$ $\frac{(n-2)\left(n^{2}-5 n+8\right)}{n^{2}}$ and $\sigma\left(P C_{n, n-2,1}\right)=\frac{2(n-2)}{n^{2}}$.

Theorem 4.3. Let $n \geq 4$ and $1 \leq p \leq n-2$. The maximal value to $\operatorname{irr}\left(P C_{n, p, 1}\right)$ is attained by $p=\frac{n-1}{2}$, if $n$ is odd, and by $p=\frac{n-2}{2}$ or $p=\frac{n}{2}$, if $n$ is even.

Proof. Under the conditions of $n$ and $p$ given by the hypothesis of the theorem, consider the graph $P C_{n, p, 1}$. From the definition of the imbalanced measure, we get $\operatorname{irr}\left(P C_{n, p, 1}\right)=2 p n-$ $2 p-2 p^{2}$. Let $f(p)=2 p n-2 p-2 p^{2}$. It also immediate to prove that the maximal value of $f(p)$ is obtained when $p=\frac{n-1}{2}$. Since $p$ is an integer number, then $n$ has to be odd. Otherwise, $f(\lceil p\rceil)=f(n / 2)=f(\lfloor p\rfloor)=f((n-2) / 2)=\left(n^{2}-2 n\right) / 2$ and the maximal value of $f(p)$ is satisfied by $f(\lceil p\rceil)$ and $f(\lfloor p\rfloor)$. 


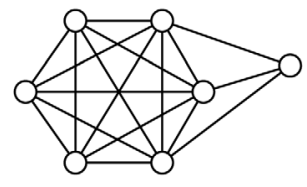

$\mathrm{PC}_{7,3,1}$

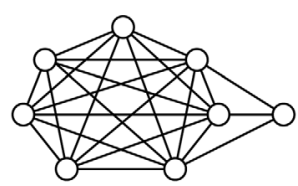

$\mathrm{PC}_{8,3,1}$

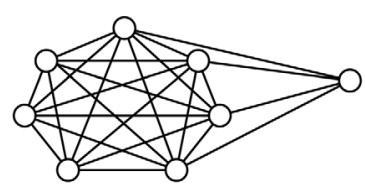

$\mathrm{PC}_{8,4,1}$

Figure $7-P C_{7,3,1}, P C_{8,3,1}$ and $P C_{8,4,1}$.

The Figure 7 shows extremal graphs $P C_{n, p, 1}$ with $n=7$ and 8 vertices, where $\operatorname{irr}\left(P C_{7,3,1}\right)=$ 18 and $\operatorname{irr}\left(P C_{8,3,1}\right)=\operatorname{irr}\left(P C_{8,4,1}\right)=24$.

Although we still have been unable to determine a simple algebraic expression for the spectral measure even for a small family of graphs such as $G=P C_{n, p, 1}$, we can show some properties of the index of this graph as illustrated in Lemmas 4.1 and 4.2 which can be helpful in the future.

Lemma 4.1. Let $n \geq 4$ and $1 \leq p \leq n-2$. The spectrum of a path complete graph $P C_{n, p, 1}$ is given by -1 with algebraic multiplicity $n-3$ and the remaining eigenvalues are the roots of the following polynomial $p(x)=x^{3}+(3-n) x^{2}-(n+p-2) x-2 p+n p-p^{2}$.

Proof. For convenient $n$ and $p$, the adjacency matrix of $P C_{n, p, 1}$ is as follows:

$$
A\left(P C_{n, p, 1}\right)=\left[\begin{array}{lll}
(J-I)_{p \times p} & J_{p \times(n-p-1)} & J_{p \times 1} \\
J_{(n-p-1) \times p} & (J-I)_{(n-p-1) \times(n-p-1)} & O_{(n-p-1) \times 1} \\
J_{1 \times p} & O_{1 \times(n-p-1)} & O_{1 \times 1}
\end{array}\right],
$$

where $J$ denotes an all-one-matrix and $I$ is the identity matrix. For $1 \leq i \leq n$, let $e_{i} \in \mathbb{R}^{n}$ be the $i$-th vector of the canonical basis. It is not too difficult to see that $\forall i, 2 \leq i \leq n-2$, if $v=e_{i}-e_{n-1}$, then $A v=-v$. So, $v$ is an eigenvector correspondent to the eigenvalue -1 with algebraic multiplicity at least $n-3$.

There is an orthogonal basis of $\mathbb{R}^{n}$ formed by the eigenvectors of $A\left(P C_{n, p, 1}\right)$. So, there are the eigenvectors as $y^{t}=(\underbrace{a, \cdots, a}, \underbrace{b, \cdots, b}, c)$. Then, the remaining 3 eigenvalues of $A\left(P C_{n, p, 1}\right)$ are the same ones of the matrix

$$
B=\left[\begin{array}{ccc}
p-1 & n-1-p & 1 \\
p & n-p-2 & 0 \\
p & 0 & 0
\end{array}\right],
$$

which are exactly the roots of $P_{B}(x)=x^{3}+(3-n) x^{2}-n x-(p-2) x-2 p+n p-p^{2}$.

Lemma 4.2. For all $p, 1 \leq p \leq n-3$, we have $\varepsilon\left(P C_{n, p, 1}\right)-\varepsilon\left(P C_{n, p+1,1}\right)=\lambda_{1}\left(P C_{n, p, 1}\right)-$ $\lambda_{1}\left(P C_{n, p+1,1}\right)+\frac{2}{n}$. 
Proof. It is well known that if $H$ is a subgraph of $G$, their indices attained the inequality $\lambda_{1}(H) \leq \lambda_{1}(G)$. The strict inequality holds if $G$ is a connected graph and $H$ is a proper subgraph of $G$. As $P C_{n, p, 1} \subset P C_{n, p+1,1}$, consequently, for $1 \leq p \leq n-3$, we have $\lambda_{1}\left(P C_{n, p, 1}\right)<$ $\lambda_{1}\left(P C_{n, p+1,1}\right)$. Let $m_{P C_{n, p, 1}}$ and $m_{P C_{n, p+1,1}}$ be the number of edges of those graphs, respectively.

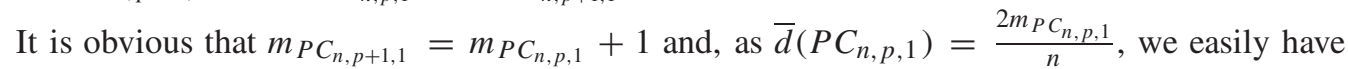
$\forall j, 1 \leq j \leq n-3, \bar{d}\left(P C_{n, j+1,1}\right)=\bar{d}\left(P C_{n, j, 1}\right)+\frac{2}{n}$. So, $\varepsilon\left(P C_{n, p, 1}\right)-\varepsilon\left(P C_{n, p+1,1}\right)=$ $\lambda_{1}\left(P C_{n, p, 1}\right)-\lambda_{1}\left(P C_{n, p+1,1}\right)+\frac{2}{n}$.

Numerically, with the aid of the software AGX, see [11], we found that the difference between the indices of the graphs $P C_{n, p+1,1}$ and $P C_{n, p, 1}$ is at most $\frac{2}{n}$. The experiments were done with graphs up to $n \leq 25$ vertices. Based on these, we establish the following conjecture.

Conjecture 4.1. For $n \geq 4$ and $1 \leq p \leq n-3$, we have $0<\lambda_{1}\left(P C_{n, p+1,1}\right)-\lambda_{1}\left(P C_{n, p, 1}\right)<\frac{2}{n}$.

From now on we will investigate the irregularity on the complete split graphs.

Definition 4.2. Let $k$ and $n$ be integer numbers such that $0 \leq k \leq n$. A graph $S(n, k)$ is a split graph if there is a partition of its vertex set into a clique of order $k$ and a stable set of order $n-k$. A complete split graph, $C S(n, k)$, is a split graph such that each vertex of the clique is adjacent to each vertex of the stable set.

The next two theorems characterize the most irregular complete split graphs relative to $s(C S(n, k))$ and $\operatorname{irr}(C S(n, k))$, respectively. As one will see, both extremal graphs are the same. Besides, the theorems show the expression of the respective measures.

Theorem 4.4. Let $k \in \mathbb{N}$ and $0 \leq k \leq n$. If $G=C S(n, k)$ is a complete split graph then $s(G)=\frac{2}{n} k(n-k)(n-1-k)$. Besides, among all complete split graphs, the most irregular one by $s(G)$ has to attend the following conditions on $k$ :

$$
k=\left\{\begin{array}{cl}
\frac{n}{3}, & \text { if } n \bmod (3)=0 ; \\
\frac{n-1}{3}, & \text { if } n \bmod (3)=1 \\
\frac{n-2}{3} \text { and } \frac{n+1}{3}, & \text { if } n \bmod (3)=2 .
\end{array}\right.
$$

Proof. Consider $G=C S(n, k)$. From the definition of the deviation measure, we have $s(G)=$ $\frac{2}{n} k(n-k)(n-1-k)$. For a given $n$, define $f(k)=\frac{2}{n} k(n-k)(n-1-k)$. The maximal value of $f(k)$ is obtained by $k=\frac{2}{3} n-\frac{1}{3} \sqrt{n^{2}-n+1}-\frac{1}{3}$. Since $k$ is an integer, we have to determine $\lceil k\rceil$ and $\lfloor k\rfloor$. Let $p, r \in \mathbb{Z}_{+}$and $0 \leq r \leq 2$ such that $n=3 p+r$. So, $n^{2}-n+1=$ $9 p^{2}+6 p r+r^{2}-3 p-r+1$. Let $r=0$, and rewrite $n^{2}-n+1=9 p^{2}-3 p+1$. Thus, $(3 p+1)^{2}-9 p=(3 p-1)^{2}+3 p$ and $3 p-1 \leq \sqrt{n^{2}+n-1} \leq 3 p+1$. Therefore, we reach $\frac{n}{3}-1<k \leq \frac{n}{3}$ if $n \bmod (3)=0$. Similarly, for $r=1$ and $r=2$, we get $\frac{n-1}{3} \leq k<\frac{n+2}{3}$, if $n$ $\bmod (3)=1$ and, $\frac{n-2}{3}<k<\frac{n+1}{2}$, if $n \bmod (3)=2$. 
From the results above, the conditions on $\lfloor k\rfloor$ and $\lceil k\rceil$ follow:

$$
\lfloor k\rfloor=\left\{\begin{array}{ll}
\frac{n}{3}-1, & \text { if } n \bmod (3)=0 ; \\
\frac{n-1}{3}, & \text { if } n \bmod (3)=1 ; \\
\frac{n-2}{3}, & \text { if } n \bmod (3)=2 ;
\end{array} \quad\lceil k\rceil=\left\{\begin{array}{cl}
\frac{n}{3}, & \text { if } n \bmod (3)=0 ; \\
\frac{n+2}{3}, & \text { if } n \bmod (3)=1 ; \\
\frac{n+1}{3}, & \text { if } n \bmod (3)=2 .
\end{array}\right.\right.
$$

Finally, after comparing $f(\lfloor k\rfloor)$ with $f(\lceil k\rceil)$ in all previous cases, we reach the following maximal values of $f(k)$,

i) $\lceil k\rceil=\frac{n}{3}$, if $n \bmod (3)=0$;

ii) $\lfloor k\rfloor=\frac{n-1}{3}$, if $n \bmod (3)=1$ and

iii) $\lfloor k\rfloor=\frac{n-2}{3}$ and $\lceil k\rceil=\frac{n+1}{3}$, if $n \bmod (3)=2$.

The Figure 8 shows extremal graphs $C S(n, k)$ with $n=5$ and 6 vertices, where $s(C S(5,1)=$ $s(C S(5,2))=4,8$ and $C S(6,2)=8$.

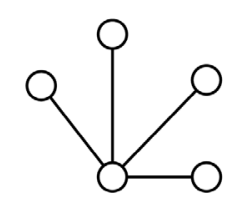

$\operatorname{CS}(5,1)=S_{5}$

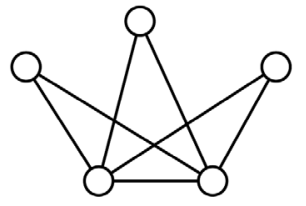

$\operatorname{CS}(5,2)$

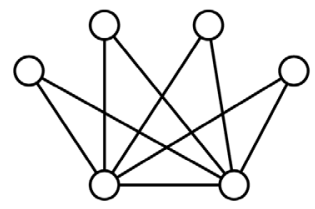

$\operatorname{CS}(6,2)$

Figure $8-C S(5,1), C S(5,2)$ and $C S(6,2)$.

Based on the results before and through the several numerical experiments done with the aid of AGX (see [14]), we propose the conjecture below.

Conjecture 4.2. Let $\mathcal{H}(n)$ be the set of all connected graphs $G$ with $n$ vertices. So, $\max _{G \in \mathcal{H}(n)}$ $s(G)=s(C S(n, k))$ where

$$
k=\left\{\begin{array}{cl}
\frac{n}{3}, & \text { if } n \bmod (3)=0 \\
\frac{n-1}{3}, & \text { if } n \bmod (3)=1 \\
\frac{n-2}{3} \text { and } \frac{n+1}{3}, & \text { if } n \bmod (3)=2 .
\end{array}\right.
$$


Theorem 4.5. Let $k \in \mathbb{N}$, and $0 \leq k \leq n$. If $G=C S(n, k)$ is a complete split graph, then $\operatorname{irr}(G)=k(n-k)(n-1-k)$. Besides, among all complete split graphs, the most irregular one relative to $\operatorname{irr}(G)$ has to satisfy the following conditions on $k$ :

$$
k=\left\{\begin{array}{cl}
\frac{n}{3}, & \text { if } n \bmod (3)=0 ; \\
\frac{n-1}{3}, & \text { if } n \bmod (3)=1 ; \\
\frac{n-2}{3} \text { and } \frac{n+1}{3}, & \text { if } n \bmod (3)=2 .
\end{array}\right.
$$

Proof. Since $C S(n, k)$ has $k$ vertices with degrees $n-1$, and $n-k$ vertices with degree $k$, we have $\operatorname{irr}(G)=k(n-k)(n-1-k)$. From Theorem 4.4, $\operatorname{irr}(C S(n, k))=\frac{n}{2} s(C S(n, k))$, and the result holds.

Among all complete split graphs relative to the variance measure, Theorem 4.6 provides the most irregular one.

Theorem 4.6. Let $k \in \mathbb{N}$ and $0 \leq k \leq n$. If $G=C S(n, k)$ is a complete split graph, we have $\sigma(S C(n, k))=\frac{1}{n^{2}}(k-n+1)^{2}(n-k) k$. Besides, among all complete split graphs, the most irregular one relative to $s(G)$ satisfies the following conditions on $k$ :

$$
k= \begin{cases}\frac{n}{4}, & \text { if } n \bmod (8)=0 \text { or } 4 \\ \frac{n-1}{4}, & \text { if } n \bmod (8)=1 \text { or } 5 \\ \frac{n-2}{4}, & \text { if } n \bmod (8)=2 \text { or } 6 \text { and } \\ \frac{n+1}{4}, & \text { if } n \bmod (8)=3 \text { or } 7\end{cases}
$$

Proof. If $G=C S(n, k)$, from the definition of the variance measure, we get $\sigma(S C(n, k))=\frac{1}{n^{2}}$ $(k-n+1)^{2}(n-k) k$. Let $n$ be given. Define $f(k)=\frac{1}{n^{2}}(k-n+1)^{2}(n-k) k$.

By elementary algebraic manipulation, we have $k=\frac{5}{8} n-\frac{1}{8} \sqrt{9 n^{2}-4 n+4}-\frac{1}{4}$ as the maximal value to $f(k)$. Similarly the proof of theorem before, the result holds.

Theorem 4.7. The eigenvalues of $C S(n, k)$ are $\frac{1}{2}(k-1) \pm \frac{1}{2} \sqrt{4 k n-2 k-3 k^{2}+1}$ each one with simple multiplicity; 0 with multiplicity $n-k-1$ and, -1 with multiplicity $k-1$.

Proof. Let $J$ be the all-one-matrix and $I$ be the identity matrix. The adjacency matrix of $C S(n, k)$ can be written as follows,

$$
A(C S(n, k))=\left[\begin{array}{cc}
(J-I)_{k \times k} & J_{k \times(n-k)} \\
J_{(n-k) \times k} & O_{(n-k) \times(n-k)}
\end{array}\right] .
$$


For $2 \leq i \leq k, v=e_{1}-e_{i}$, where $e_{i}$, is the $i$-th vector of the canonical basis, is an eigenvector of $A(C S(n, k))$ corresponding to the eigenvalue -1 . Its multiplicity is at least $k-1$. Besides, note that 0 is an eigenvalue of $A(S C(n, k))$ to the eigenvectors $x=e_{j}-e_{n}, k+2 \leq j \leq n$. In the last case, its multiplicity is at least $n-k-1$. The matrix $A(C S(n, k))$ has an eigenvector with the form $y^{t}=(\underbrace{a, \cdots a,} \underbrace{b, \cdots b})$. Thus, we conclude that their remaining 2 eigenvalues are equal to the ones of

$$
B=\left[\begin{array}{cc}
k-1 & n-k \\
k & 0
\end{array}\right] .
$$

Consequently, $\frac{1}{2}(k-1) \pm \frac{1}{2} \sqrt{4 k n-2 k-3 k^{2}+1}$ are eigenvalues of $A(C S(n, k))$.

From Theorem 4.7, the spectral measure of complete split graphs with $n$ vertices and a clique of size $k$ is

$$
\varepsilon(C S(n, k))=\frac{1}{2 n}\left(2 k-n-3 k n+2 k^{2}+n \sqrt{4 k n-2 k-3 k^{2}+1}\right) .
$$

Although Theorem 4.7 has characterized the eigenvalues to any complete split graph, three of them were implicitly determined. It follows that, the maximum of the spectral measure function is not a simple task. The numerical experiments with done (see [14]) show that, among all connected graphs with $n$ vertices, the complete split graphs are the most irregular to the spectral measure. More specifically, they are the stars $S_{n}=C S(n, 1)$, for $4 \leq n \leq 11$, and $C S(n, 2)$, for $12 \leq n \leq 15$.

Tables 2 and 3 summarize our results relative to extremalities concerning $P C_{n, p, 1}$ and $S C(n, k)$ graphs respectively.

Table 2 - The most irregular path complete graphs to $s(G), \operatorname{irr}(G), \sigma(G)$ and $\varepsilon(G)$.

\begin{tabular}{|l|l|l|}
\hline \multicolumn{1}{|c|}{ Measures of irregularity } & Extremal graphs in $P C_{n, p, 1}$ & References \\
\hline$s\left(P C_{n, p, 1}\right)$ & $P C_{n, 1,1}$ & Theorem 4.1 \\
$=\frac{1}{n}((n-p-1)(n+2 p-2+|n-2 p-2|)$ & & \\
\hline$\sigma\left(P C_{n, p, 1}\right)$ & $P C_{n, 1,1}$ & Theorem 4.2 \\
$=\frac{1}{n^{2}}((n-p-1)[(n-4)(n-p)+4])$ & $P C_{n, \frac{n-1}{2}, 1}$ if $n$ is odd; & Theorem 4.3 \\
\hline $\operatorname{irr}\left(P C_{n, p, 1}\right)$ & $P C_{n, \frac{n}{2}, 1}$ or $P C_{n, \frac{n-2}{2}, 1}$ if $n$ is even. & \\
$=k(n-k)(n-1-k)$ & $P C_{n, 1,1}$ & Conjecture 4.1 \\
\hline$\varepsilon\left(P C_{n, p, 1}\right)$
\end{tabular}


Table 3 - The most irregular split complete graphs to $s(G), \operatorname{irr}(G), \sigma(G)$ and $\varepsilon(G)$.

\begin{tabular}{|c|c|c|}
\hline Measures of irregularity & Extremal graphs in $S C(n, k)$ & References \\
\hline $\begin{array}{l}s(S C(n, k)) \\
=\frac{2}{n} k(n-k)(n-1-k)\end{array}$ & $k=\left\{\begin{array}{l}\frac{n}{3}, \quad \text { if } n \bmod (3)=0 \\
\frac{n-1}{3}, \quad \text { if } n \bmod (3)=1 \\
\frac{n-2}{3} \text { and } \frac{n+1}{3}, \quad \text { if } n \bmod (3)=2 .\end{array}\right.$ & Theorem 4.4 \\
\hline $\begin{array}{l}\operatorname{irr}(S C(n, k)) \\
=k(n-k)(n-1-k)\end{array}$ & $k=\left\{\begin{array}{l}\frac{n}{3}, \text { if } n \bmod (3)=0 \\
\frac{n-1}{3}, \quad \text { if } n \bmod (3)=1 \\
\frac{n-2}{3} \text { and } \frac{n+1}{3}, \text { if } n \bmod (3)=2\end{array}\right.$ & Theorem 4.5 \\
\hline $\begin{array}{l}\sigma(S C(n, k)) \\
=\frac{1}{n^{2}}(k-n+1)^{2}(n-k) k\end{array}$ & $k= \begin{cases}\frac{n}{4}, & \text { if } n \bmod (0)=0 \text { or } 4 \\
\frac{n-1}{4}, & \text { if } n \bmod (8)=1 \text { or } 5 \\
\frac{n-2}{4}, & \text { if } n \bmod (8)=2 \text { or } 6 \\
\frac{n+1}{4}, & \text { if } n \bmod (8)=3 \text { or } 7 .\end{cases}$ & Theorem 4.6 \\
\hline $\begin{array}{l}\varepsilon(S C(n, k)) \\
=\frac{1}{2 n}\left(2 k-n-3 k n+2 k^{2}\right. \\
\left.\quad+n \sqrt{4 k n-2 k-3 k^{2}+1}\right)\end{array}$ & $\begin{array}{l}S_{n}=S C(n, 1), \text { if } 4 \leq n \leq 11 \\
S_{n}=S C(n, 2), \text { if } 12 \leq n \leq 15\end{array}$ & Tests AGX, [14] \\
\hline
\end{tabular}

\section{CONCLUSION}

Among all graphs with $n$ vertices and concerning all the measures of irregularity investigated, the quasi complete graphs are amongst the most irregular ones. However, such an observation is not enough to allow us to identify which irregularity measure is more accurate. Therefore, we have decided to investigate the irregularity in more restricted classes of graphs, such as path complete graphs $P_{n, p, t}$ (in $t=1$ cases) and complete split graphs $S C(n, k)$, where $k$ is the size of the maximal clique of the graph (see Section 4). This investigation allows us to state that:

(i) as it was said before, the imbalance measure does not satisfy the definition of the function $F$ given in Introduction. In fact, in the particular case of $P C_{n, p, 1}$ graphs, the behavior of the invariant $\operatorname{irr}\left(P C_{n, p, 1}\right)$ does not work as it would be expected (see the third line of Table 2);

(ii) the other three measures, $\varepsilon(G), s(G)$ and $\sigma(G)$, are not comparable. In other words, none of them is more accurate that the other.

\section{ACKNOWLEDGMENTS}

Supported by CNPq-Brazil grants 141904/2008-9, 303456/2009-4, 305516/2010-8 and 305016/2006-7. 


\section{REFERENCES}

[1] Ahlswede R \& Katona GOH. 1978. Graphs with Maximal Number of Adjacent Pairs of Edges. Acta Mathematica Academice Scientiarum Hungaricae, 32: 97-120.

[2] Albertson P. 1997. The Irregularity of a graph. Ars Combinatoria, 46: 219-225.

[3] Aouchiche M, Bell F, Cvetković D, Hansen P, Rowlinson P, Simiki SK \& Stevanović D. 2008. Variable neighborhood search for extremal graphs. 16. Some conjectures related to the largest eigenvalue of a graph. European Journal of Operational Research, 191(3): 661-676.

[4] BELL FK. 1990. On the maximal index of connected graphs. Linear Algebra and its Applications, 161: $45-54$.

[5] Belhaiza S, Hansen P, Abreu NMM \& Oliveira CS. 2005. Variable Neighborhood Search for Extremal Graphs. XI. Bounds on Algebraic Connectivity. Graph Theory and Combinatorial Optimization, Spring, 1-16.

[6] BRandstÄDt A, LE VB \& SPIndRad JP. 1999. Graph Classes: a Survey. SIAM Monographs on Dioscrete Mathematics and Applications.

[7] BRualdi RA \& Solheid ES. 1986. On the spectral radius of connected graphs. Publications de L'Institut Mathématique (Beograd) (N.S.), 39(53): 45-54.

[8] Collatz L \& Sinogowitz U. 1957. Spektren endlicher grafen. Abh. Math. Sem. Univ. Hamburg, 21: $63-77$.

[9] Cvetković D \& Rowlinson P. 1988. On connected graphs with maximal index. Publications de L'Institut Mathématique (Beograd) (N.S.), 44(58): 29-34.

[10] Hansen P \& MÉlot H. 2005. Variable Neighborhood Search for Extremal Graphs 9. Bounding the Irregularity of a Graph. American Mathematical Society, 69: 253-264.

[11] Hansen P \& Caporossi G. 2000. AutoGraphiX: An Automated System for Finding Conjectures in Graph Theory. Electronic Notes in Discrete Mathematics, 5: 158-161.

[12] Harary F. 1962. The maximum connectivity of graphs. Proc. Nat. Acad. Sci. U.S.A., 48: 11421146.

[13] Nikiforov V. 2006. Eigenvalues and degree deviation in graphs. Linear Algebra and its Applications, 414: 347-360.

[14] Oliveira JA. 2012. Medidas de Irregularidade em Grafos. Tese de Doutorado, COPPE/UFRJ. 